Revista Mexicana de Análisis de la Conducta - Mexican Journal of Behavior Analysis 2017 | Núm. 1 • junio | No. 1 • June | Vol. 43, 60-82 |

DOI: http://dx.doi.org/10.5514/rmac.v43.11.61076

\title{
COMPARACIÓN DE MÉTODOS DE CORRECCIÓN DE ERRORES EN LA ADQUISICIÓN DE DISCRIMINACIONES CONDICIONALES
}

\section{COMPARISON OF ERROR CORRECTION TRIALS IN CONDITIONAL DISCRIMINATIONS ACQUISITION}

\author{
María Ruiz Pérez, Javier Quesada Amor, Andrés García García \& María \\ Teresa Gutiérrez Domínguez \\ Universidad de Sevilla
}

\section{Resumen}

El objetivo del estudio fue comparar diferentes métodos de corrección de errores en una tarea de discriminación condicional, en participantes de diferentes grupos de edad (7-8, 10-11, 15-16 y 19-20 años), para determinar el tipo de corrección más eficaz en el aprendizaje. Se diseñó un experimento en el que se usó una tarea de igualación a la muestra de primer orden. En la tarea, cuatro letras latinas funcionaron como estímulo muestra, y cuatro símbolos chinos funcionaron como estímulos de comparación. En caso de error en la respuesta de los participantes, se utilizó uno de los tipos de corrección contemplados en esta investigación: castigo positivo, seguimiento de regla, corrección llevada a la muestra y corrección llevada a las comparaciones. Además, se contó con un grupo control, que no recibió ninguna corrección ante los errores. Se contabilizó el número de ensayos necesarios para

María Ruiz Pérez y Javier Quesada Amor, Sevilla, España; Andrés García García y María Teresa Gutiérrez Domínguez, Departamento de Psicología Experimental, Universidad de Sevilla.

Los autores agradecen a José Antonio Moreno Martínez y Macarena Pérez Porrero su colaboración en la búsqueda de participantes para llevar a cabo esta investigación.

Enviar correspondencia a la primera autora: maria_rp91@hotmail.com 
aprender la tarea. Los resultados mostraron diferencias entre los grupos que recibieron corrección con respecto al que no, siendo la ejecución peor en este último. Además, se encontró que la corrección con castigo positivo ralentizó el aprendizaje de la tarea. Los resultados resaltan la importancia del uso de corrección diferente del castigo positivo en las tareas de discriminación condicional.

Palabras clave: discriminación condicional, corrección, velocidad de adquisición, aprendizaje

\begin{abstract}
The aim of the study was to compare different methods of error correction in a conditional discrimination task presented to subjects of different age groups (7-8, 10-11, 15-16 and 19-20 years) to determine the most effective type of learning correction by this method. A first-order matching-to-sample task was used. In this task four Latin letters functioned as sample stimuli, and four Chinese symbols functioned as comparison stimuli. Whenever an error occurred, one of four types of correction was used: positive punishment, rule following, correction applied to sample and correction applied to comparisons. In addition, participants in a control group did not receive any correction following errors. The number of trials required for each participant to learn the task was analyzed. Differences in the execution between the groups that received different correction procedures revealed that performance was worse in the group that did not receive correction. Additionally, the number of trials required to learn the task was higher with positive punishment. The results emphasize the importance of use of correction procedures in conditional discrimination tasks.

Keywords: conditional discrimination, correction, acquisition speed, learning

El papel de las discriminaciones en los contextos de aprendizaje es de suma importancia debido a que proporcionan a las personas el medio para adquirir un alto número de habilidades, es decir, la capacidad de actuar de manera eficaz en un tipo determinado de situación. Se aprende a emitir conductas determinadas ante estímulos concretos, de forma que lo importante de un comportamiento es su función, la cual está determinada por el contexto, por las alteraciones en éste y por los cambios que el individuo genera en él (Pérez, 2001).
\end{abstract}


La mayor parte de discriminaciones que ocurren a lo largo de la historia de aprendizaje de un individuo son condicionales (Pérez, 2001). Una discriminación condicional es una unidad de cuatro términos (Sidman, 2000) en la que la presencia del estímulo condicional va a supeditar la relación existente entre los tres elementos restantes. Dentro de este tipo de discriminaciones, el procedimiento más utilizado es la igualación a la muestra (García, 2012), en la que el estímulo condicional será conocido como muestra y los discriminativos y deltas como comparaciones (Rodríguez, García, Gutiérrez, Pérez, \& Bohórquez, 2009).

$\mathrm{Al}$ ser las discriminaciones condicionales, como ya se ha comentado, un medio para llegar a alcanzar un alto número de habilidades, resulta interesante el estudio de las variables que puedan ejercer influencia en la adquisición de éstas de una manera más rápida. Además, es conveniente señalar que son la base de otro procedimiento de mayor complejidad: las clases de equivalencia, que fueron identificadas inicialmente por Sidman en el año 1971 y que más adelante fueron especificadas sus propiedades esenciales (reflexividad, simetría y transitividad) por Sidman y Tailby (1982). El atractivo de este fenómeno radica en la derivación de relaciones que no se han entrenado de manera expresa (Pérez, 2015).

En la literatura se han experimentado con diferentes variables que afectan a la ejecución de los participantes en las discriminaciones condicionales. Por ejemplo, el momento de presentación del reforzador, que fue estudiado por Ribes y Martínez (1990), la naturaleza del mismo (visual, auditiva y táctil) (Ortega, Pacheco, Sánchez, \& Rivera, 2012), la duración del intervalo entre ensayos (Flores \& Bruner, 2005) o el papel de las instrucciones proporcionadas (Martínez, Ortiz, \& González, 2007; Martínez \& Tamayo, 2005; Ortiz \& Cruz, 2011).

Sin duda, uno de los factores más importantes en este tipo de tarea es la retroalimentación proporcionada a los participantes, la cual puede entenderse como el proceso por el que se da a conocer al individuo cómo ha sido su ejecución según sus resultados (Islas \& Flores, 2007). Existen multitud de trabajos que intentan conocer el papel de la retroalimentación en discriminaciones condicionales centrándose en estudiar como variable experimental el tipo y la manera en la que se administra un reforzador ante un acierto. Véase por ejemplo los trabajos de García et al., (2011) y Ortega et al., (2012) o la revisión bibliográfica sobre esta temática que puede encontrarse en el artículo de Villanueva, Mateos y Flores (2008). Sin embargo, en cuanto a consecuencias proporcionadas cuando se comete un error en una tarea de discriminación condicional, como ya apuntaban Valero y Luciano (1997), existen pocos estudios en la literatura científica que investiguen los efectos 
de la aplicación de estas contingencias diferenciales durante un entrenamiento en igualación a la muestra.

La retroalimentación que se proporciona a modo de consecuencia cuando un participante emite una respuesta errónea, y cuya finalidad es hacer disminuir la probabilidad de aparición de la conducta a la que sigue, es lo que en este trabajo es considerado como corrección. Normalmente, en los primeros trabajos realizados se aplicaban distintos reforzadores cuando se producía una respuesta adecuada, pero no se solía mencionar de forma manifiesta qué tipo de corrección se suministraba en caso de fallo. En los pocos estudios en los que se explican las contingencias realizadas, éstas no han sido investigadas sistemáticamente como un aspecto que pueda influir en el aprendizaje de la tarea en cuestión (Valero \& Luciano, 1997).

En trabajos como los de Sidman y Cresson (1973), Sidman y Talby (1982) y Constantine y Sidman (1975) se exponen algunos métodos de corrección de errores que sí fueron tomados como una variable independiente en la investigación (continuidad en el ensayo hasta que ocurriera el acierto, vuelta atrás en la tarea $o$ incremento del tiempo del intervalo entre ensayos). Del mismo modo se muestran métodos de corrección en el trabajo de Rodgers y Iwata (1991), que comparan tres condiciones experimentales de corrección de errores: la condición de línea base, donde se proporciona un reforzamiento diferencial, la condición de práctica, donde el error fue seguido de la repetición del ensayo hasta que se dio la respuesta correcta y la condición de escape, donde el error fue seguido de ensayos con estímulos irrelevantes (igualación de colores).

También es el caso del estudio de Worsdell et al., (2005), quienes realizaron varios experimentos para determinar qué manera de corregir errores aceleraba más el aprendizaje. En el primer experimento comparan la repetición simple del ensayo (una vez) y la repetición múltiple (cinco veces). En el segundo comparan la repetición múltiple del ensayo de forma continua (cada vez que se comete un error) y la repetición intermitente (cada tres errores). En el último de los experimentos se compara la eficacia de la repetición múltiple con una palabra relevante para la tarea (la misma que se les muestra en el ensayo) o irrelevante (que no aparece durante el entrenamiento). Estos autores encuentran que se optimiza el aprendizaje con repeticiones múltiples implementadas de manera continua y con palabras relevantes para la tarea.

Actualmente, se pueden encontrar experimentos en los que se aplica algún tipo de retroalimentación cuando la respuesta que emite el participante no es la adecuada, pero estas correcciones siguen sin tomarse como un aspecto a estudiar de 
manera aislada. El procedimiento que se suele emplear en los trabajos con animales no humanos cuando éstos cometen un error es el de corrección llevada a la muestra, o bien, llevada a las comparaciones. Esto consiste en la presentación de un tiempo fuera y el consiguiente retorno al ensayo en el que se ha errado, presentándolo desde el estímulo de muestra en el caso de corrección llevada a la muestra o desde la pantalla con la muestra y las comparaciones en el caso de corrección llevada a las comparaciones. Trabajos con palomas que usan dicha forma de proceder son los de García y Benjumea (2006) y Gómez, García y Pérez (2014).

Otro de los procedimientos que se puede usar como corrección es el castigo positivo, descrito por Skinner (1938) cuando, observó que la presencia de un estímulo aversivo producía una disminución de la conducta operante con la cual se correlacionaba, aunque bien es cierto que el beneficio de este modo de actuar no está bien fundamentado. Los experimentos de Thorndike (1906) mostraron que las respuestas cuya consecuencia era el anuncio de que se había cometido un error no descendían, mientras que las respuestas seguidas del anuncio de acierto sí incrementaban su ocurrencia. El propio Skinner (1953), en "Ciencia y Conducta Humana", pone en duda los efectos del castigo y señala limitaciones y efectos secundarios que presenta, como el efecto temporal y el desarrollo de emociones negativas generalizadas. Asimismo, el castigo tiene efectos circunscritos a la situación o persona que lo aplica, por lo que los aprendizajes logrados mediante este método son difícilmente extrapolables a otros contextos (Kazdin, 1994). A pesar de ello, es la variable más usada cuando un participante falla en una tarea de igualación a la muestra; por ejemplo, los experimentos de Hurtado, Robayo y Peña (2007), Ortiz y Cruz (2011), Rodríguez, Ribes, Valencia y González (2011) o Serrano y Montes (2014).

Por otra parte, en la enseñanza aplicada a población con necesidades educativas especiales, se suele utilizar otro tipo de corrección que consiste en la repetición del ensayo, presentando nuevamente los estímulos antecedentes junto a una ayuda que facilita la respuesta correcta (Greer \& Ross, 2014). Esta modalidad de corrección se puede aplicar con diferentes grados de intrusividad y complejidad, que pueden ir desde una ayuda total a la emisión de una instrucción verbal. El objetivo es instigar la realización de una conducta concreta que puede ser de interés especial para el instructor en la realización de una tarea (Barón \& Galizio, 1990).

Lo relevante para este estudio sobre dicha manera de corregir es el seguimiento de una regla o instrucción, lo cual se entiende como una descripción verbal que especifica las contingencias pasadas o futuras de un individuo (Ortiz \& Cruz, 2011). No obstante, debido a que la implicación de conducta verbal complejiza el procedimiento y, para no desviar la atención del objetivo de investigación principal, se 
entiende el seguimiento de regla como la presentación de un estímulo discriminativo que hace más probable la aparición de una conducta concreta que es relevante para el aprendizaje de la tarea. Esto puede entenderse así, dado que determinados antecedentes pueden lograr adquirir la función de normas o instrucciones que, actuando como estímulos discriminativos, ejercen control sobre la conducta sin la intervención de forma manifiesta de contingencias moldeadoras (Törneke, Luciano, \& Valdivia, 2008).

A pesar de ser topográficamente diferente, un estímulo discriminativo no verbal puede llegar a ser funcionalmente equivalente a una regla verbal explícitamente formulada. Para ello es necesario un entrenamiento en múltiples ejemplares que permita generalizar relaciones, de forma que, el individuo aisle las claves contextuales de una situación para aplicarlas a una nueva (Quesada, García, Ruiz, \& García, 2015). Por tanto, es posible que esta capacidad, esté más desarrollada en una población que tenga un mayor bagaje comportamental en relación con esto.

Con base en lo expuesto sobre las formas de corrección y la carencia de trabajos que abordan esta variable de manera empírica, este estudio sugiere que pueden existir diferencias en la velocidad con la que se alcanza un aprendizaje concreto según el tipo de procedimiento correctivo que se administre. Bajo esta premisa, el presente trabajo plantea como objetivo principal la comparación entre diferentes formas de corrección de errores (castigo positivo, seguimiento de una regla, corrección llevada a la muestra y llevada a las comparaciones) con el fin de determinar cuál de es más eficaz a la hora de adquirir una respuesta mediante una tarea de discriminación condicional y observar, además, la ejecución de la tarea en diferentes grupos de edad (7-8, 10-11, 15-16 y 19-20 años). Esto posee especial interés debido a las consecuencias prácticas que se pueden derivar de los resultados que arroje esta investigación, repercutiendo todo esto en una aceleración del aprendizaje que confluye en una mejora de la práctica educativa.

Para dar respuesta a este planteamiento, se proponen como hipótesis de investigación, por un lado, que la corrección por seguimiento de reglas será más efectiva en los participantes mayores que en los de menores y, por otro, que la corrección con castigo positivo será menos efectiva que el resto.

\section{Método}

\section{Participantes}

El total de la muestra estuvo formada por 96 personas de ambos sexos (53 hombres y 43 mujeres), residentes en la provincia de Sevilla, todas ellas de desarrollo 
Tabla 1

Participantes en función de la edad y el sexo

\begin{tabular}{lcccc}
\hline & $7-8$ años & $10-11$ años & $15-16$ años & $19-20$ años \\
\hline Hombre & 13 & 15 & 13 & 12 \\
Mujer & 12 & 7 & 11 & 13 \\
\hline
\end{tabular}

típico. La edad de los participantes osciló entre los siete y los veinte años. La participación fue voluntaria y, en caso de los menores de edad, se contó con el consentimiento de los tutores legales. La elección de los participantes se llevó a cabo mediante un muestreo por accesibilidad. La distribución de los participantes en función del sexo y la edad se muestra en la Tabla 1.

\section{Materiales}

Para llevar a cabo el experimento fue necesario un ordenador portátil, el cual poseía la base de datos Microsoft Office Access Database con el diseño específico de la tarea de igualación a la muestra de primer orden a presentar, además de un ratón con el que los participantes realizaron el experimento.

Los estímulos que se usaron en la tarea se exponen en la Figura 1. Los estímulos empleados como estímulos de muestra fueron cuatro letras latinas, los estímulos de comparación los conformaban cuatro caracteres chinos y se utilizaron "emoticonos" felices y tristes como retroalimentación positiva y negativa respectivamente.

\section{Procedimiento}

Las sesiones de recogida de datos se realizaron en una sala habilitada a tal efecto y alejada de ruidos. Una vez ahí, se efectuaba con los participantes el procedimiento de consentimiento informado (en caso de ser menores de edad se hacía con los padres o tutores legales). Después, se les asignaba aleatoriamente una condición entre todas las posibles y se pasaba a la situación experimental, donde las personas se sentaban delante de la pantalla del ordenador y se les proporcionaban las instrucciones, que fueron las mismas para los cuatro grupos de edad:

Ahora se te va a presentar una tarea en la que tendrás que elegir una letra china dependiendo de la letra española que se te muestre. Podrás utilizar el ratón para seleccionar la opción que quieras. Si tienes alguna duda durante la prueba se te responderá cuando finalices. Ahora te voy a hacer un ejemplo para que veas cómo es. 


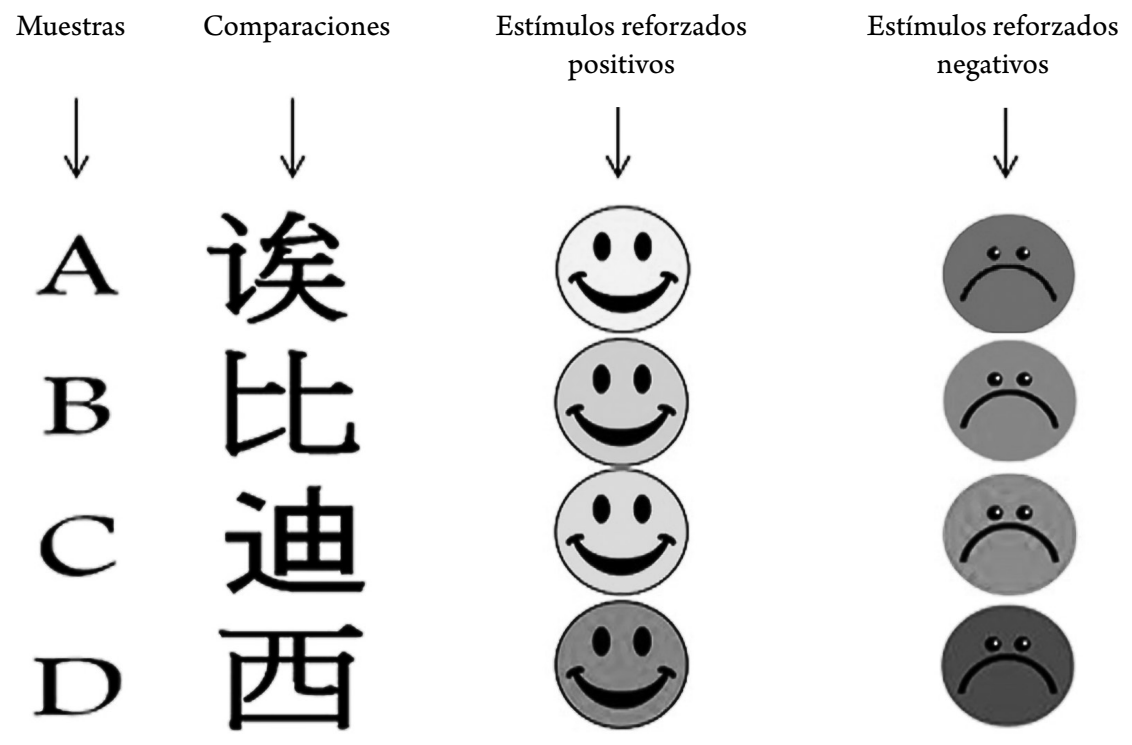

Figura 1. Estímulos utilizados en la tarea experimental. Cuatro letras latinas que funcionaron como estímulo de muestra, cuatro caracteres chinos que actuaron como estímulos de comparación, cuatro emoticonos alegres y otros cuatro tristes que se utilizaron como estímulos reforzadores positivos y negativos respectivamente.

A continuación, el investigador procedía a realizar un ensayo en forma de guía: En el centro de la pantalla en blanco aparecía una imagen cuadrada $(7 \times 7 \mathrm{~cm})$ con una letra latina (como se observa en la Figura 2) en la cual se pinchaba y aparecían, con una demora de 0 segundos, las cuatro teclas $(7 \times 7 \mathrm{~cm})$ con los estímulos de comparación (véase Figura 3). Entonces se le decía al participante que debía escoger la que creyese oportuna. Se le pasaba el ratón, se le dejaba que seleccionara una de las opciones de comparación y a partir de ahí tenía que realizar de manera individual el resto de ensayos del bloque, que constó de un total de 192, con un intervalo entre ensayos de 5 segundos.

Los estímulos que se mostraban en la pantalla fueron presentados de forma contrabalanceada a lo largo de toda la tarea experimental, de forma que, ante cada muestra, las comparaciones se mostraban en todas las posiciones el mismo número de veces, evitando así la preferencia del participante por una u otra ubicación.

En todas las condiciones del experimento, al seleccionar la opción correcta, se pasaba durante dos segundos a una pantalla en blanco con una imagen central de un 


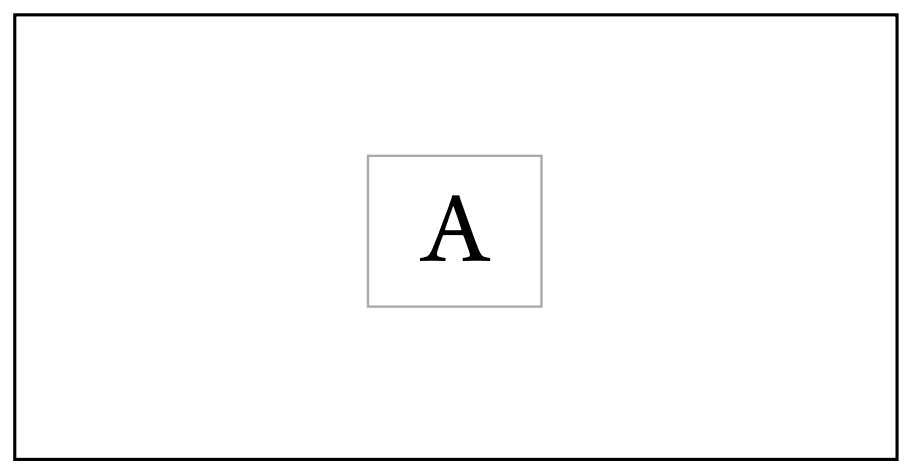

Figura 2. Ejemplo de la pantalla con el estímulo de muestra que se les presentaba a los participantes. Esta imagen fue la misma para cada una de las condiciones (la letra que aparecía fue variando de un ensayo a otro). Los participantes debían hacer click en ella para que apareciesen las comparaciones.

emoticono alegre ( $7,5 \mathrm{~cm}$ de radio), que cambiaba de color aleatoriamente en sus diferentes presentaciones. Al finalizar estos dos segundos se presentaba el intervalo entre ensayos y se continuaba con la tarea.

En el caso de error en la respuesta del participante, se procedía a proporcionar las contingencias dependiendo de la condición que se le hubiese asignado con anterioridad. Para el grupo control (sin corrección), al responder de manera incorrecta se pasaba directamente al siguiente ensayo. En el caso de la corrección con castigo positivo, al fallar, se presentaba una pantalla en blanco con un emoticono triste (6 $\mathrm{cm}$ de radio) en el centro durante dos segundos, que cambiaba de color aleatoriamente en sus diferentes presentaciones. Después de la aparición de éste estímulo se continuaba con el siguiente ensayo. En la condición de seguimiento de regla, al errar, la opción correcta se enmarcaba en negro y comenzaba a parpadear. No se pasaba al siguiente ensayo hasta que no se seleccionaba la alternativa apropiada. Cuando se empleaba corrección llevada a la muestra, se aplicaba un tiempo fuera de dos segundos y a continuación se volvía a la muestra de ese mismo ensayo, mientras que, cuando se utilizaba corrección llevada a las comparaciones (quinta y última condición), después del tiempo fuera se volvía a la pantalla donde estaban las cuatro comparaciones y la muestra. En estos dos últimos casos no se pasaba al siguiente ensayo hasta que no se proporcionaba la respuesta adecuada.

Los participantes realizaban la tarea hasta que, bien alcanzasen el criterio establecido de 12 ensayos consecutivos realizados correctamente, o bien finalizasen el 


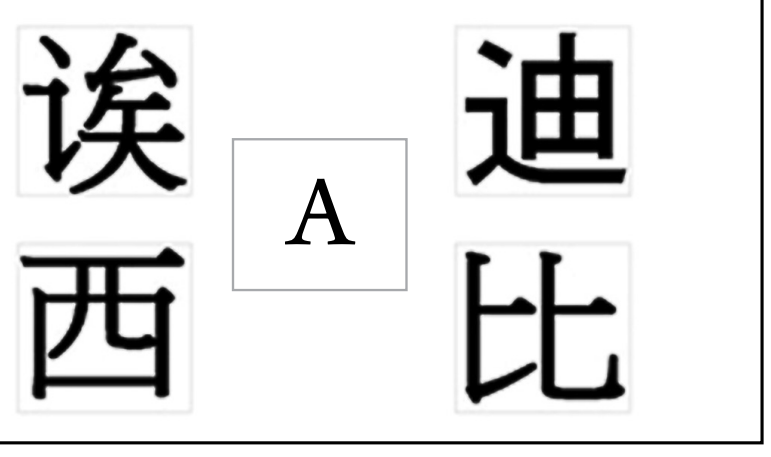

Figura 3. Ejemplo de pantalla donde se puede ver el estímulo de muestra en el centro junto a las cuatro comparaciones que aparecían una vez ocurría la respuesta de observación. Los estímulos utilizados como comparación fueron los mismos en todas las condiciones experimentales y la posición de estos fue variando en los diferentes ensayos de forma contrabalanceada.

bloque de 192 ensayos que conforman la tarea experimental. Al darse una de estas dos situaciones, la recogida de datos se daba por concluida.

El programa en el que se realizó la tarea cumplía las funciones de presentación de estímulos, registro de respuestas, presentación del tipo de corrección en caso de fallo y señalización del fin de la prueba.

\section{Diseño}

El diseño de este estudio fue factorial $5 \times 4$ univariado. Contó con tres variables: Dos variables independientes y una variable dependiente. La primera de las variables independientes fue el tipo de corrección (VI1), que tuvo los siguientes valores: sin corrección (control), castigo positivo, seguimiento de regla, corrección llevada a la muestra y corrección llevada a las comparaciones. La segunda de ellas era la edad (VI2), a la cual se le asignaron cuatro valores: 7-8 años, 10-11 años, 15-16 años y 19-20 años. La variable dependiente (VD) que se contempló en esta investigación fue la velocidad de adquisición de discriminaciones condicionales, que se midió con el número de ensayos que cada participante necesitó para adquirir el criterio (12 ensayos correctos consecutivos).

La estrategia de recogida de datos fue mixta, ya que los datos del entrenamiento de los diferentes grupos fueron recogidos de manera longitudinal en distintos momentos (ensayos) y las dos variables independientes fueron registradas de manera transversal. 


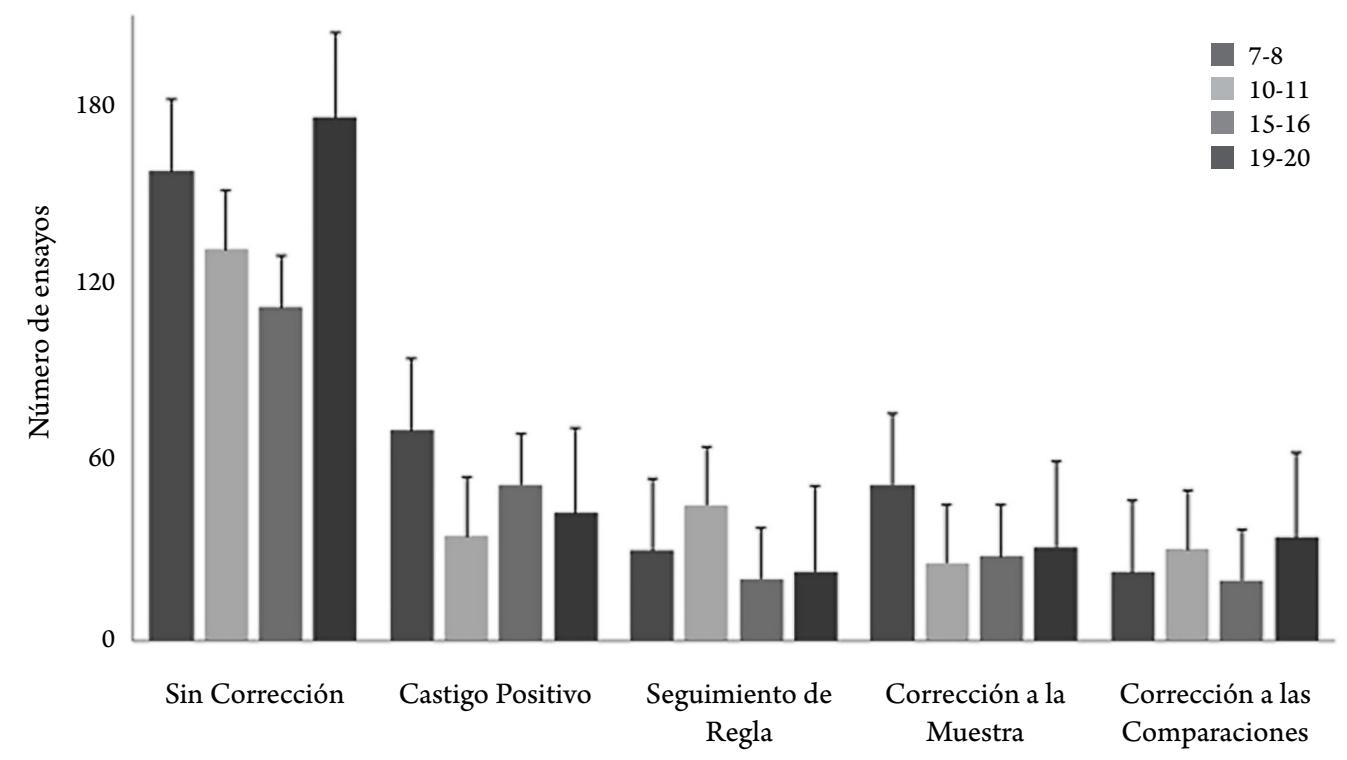

Figura 4. Diferencias en el número promedio de ensayos necesarios para alcanzar el criterio de aprendizaje en la tarea. Las medias están agrupadas por edad así como por tipo de corrección. La diferencia de medias entre el grupo control y los experimentales fue estadísticamente significativa.

\section{Análisis de datos}

Para el análisis estadístico se usó el paquete Statistical Package for the Social Sciences (SPSS Statistics, versión 19). Se realizaron análisis con ANOVA factorial, ANOVA de un factor y $F$ de Welch, con un nivel de significación de .05 para cada una de las hipótesis. Previamente, se contrastó el cumplimiento de homocedasticidad mediante la prueba $\mathrm{F}$ de Levenne ( $\mathrm{p}>.05$ ), usando como alternativa heterocedástica la prueba $\mathrm{F}$ de Welch en caso de incumplimiento.

\section{Resultados}

Se pueden observar diferencias en el número de ensayos necesarios para alcanzar el criterio de aprendizaje de la tarea según si se proporciona retroalimentación o no (véase Figura 4$)$, siendo el grupo control $(M=144.28, D T=69.17)$ el que mayor número de ensayos ha precisado, además de presentar un elevado porcentaje de participantes que no lograron aprender la tarea (66.7\%). Dentro de los grupos experimentales, los participantes que recibieron corrección con castigo positivo ( $M$ 
$=50.15, D T=35.54)$ han sido los que han tenido una peor ejecución, mientras que la corrección llevada a las comparaciones $(M=27.01, D T=10.00)$ ha sido la que mejores resultados ha arrojado.

En lo referente a la edad, los participantes del grupo de 7-8 años $(M=66.72$, $D T=65.41)$ son los que han requerido más ensayos en el aprendizaje de la tarea en contraposición al grupo de $15-16$ años $(M=43.92, D T=47.94)$, que presenta la media más baja entre los cuatro rangos de edad estudiados.

En el ANOVA factorial desarrollado, se encontraron diferencias estadísticamente significativas en el tipo de corrección proporcionada a los participantes, dando lugar a un tamaño de efecto grande, $F(4,75)=43.29, p=.000, R^{2}=.65$. Dado que, tanto la significación como el tamaño de efecto apuntan en la misma dirección, es probable que los datos encontrados se encuentren también a nivel poblacional.

En la prueba de comparaciones múltiples a posteriori de Tukey se detectó como significativa ( $p s=.000$ en todos los casos) la diferencia de medias entre el grupo sin corrección y el resto de grupos de esa variable. Ninguna otra comparación resultó estadísticamente significativa.

Por otro lado, la cantidad de ensayos necesarios para completar la tarea no fue significativamente diferente en función de la edad de los participantes, $F(3,75)$ $=1.12, p=.348, R^{2}=.06$. No obstante, el tamaño de efecto encontrado fue medio. El resultado es poco concluyente, ya que los índices no señalan de manera clara hacia un mismo sentido.

Asimismo, la interacción entre el tipo de corrección y la edad tampoco alcanzó la significación estadística. Sin embargo, el tamaño de efecto fue grande, $F(12,75)$ $=1.04, p=.424, R^{2}=.12$. Con mayor potencia estadística debida a un mayor tamaño muestral, el análisis planteado podría haber llegado a alcanzar la significación estadística, por lo que los resultados obtenidos no son concluyentes.

\section{Efectividad de la corrección por seguimiento de regla en los participantes mayores y en los menores}

La diferencia de medias entre el grupo de edad de 7-8 años y el de 19-20 en la corrección por seguimiento de regla es reducida, aunque es levemente mayor la del grupo de menor edad $(M=30.20, D T=14.41)$ que la del grupo con el rango de edad más alto $(M=23.20, D T=6.46)$.

La comparación a priori realizada mediante ANOVA de un factor entre el grupo de 7-8 y 19-20 años no resultó estadísticamente significativa y tuvo un tamaño de efecto medio, $t(16)=.92, p($ una cola $)=.187, R^{2}=.05$. Los resultados parecen su- 
gerir que la hipótesis nula es verdadera y, por tanto, las diferencias que se observan en la gráfica pueden ser explicadas por el azar.

\section{Efectividad de la corrección con castigo positivo respecto al resto}

Se observan diferencias (Figura 5) en el número de ensayos realizados en función del tipo de corrección aplicada. El grupo al que se administró corrección con castigo positivo $(M=42.69, D T=12.49)$ presenta la media más alta. La más baja y, por tanto, más efectiva fue la corrección llevada a las comparaciones $(M=29.90$, $D T=10.00)$.

En los contrastes a priori llevados a cabo a través de la prueba F de Welch se encontraron diferencias estadísticamente significativas entre el grupo corregido con castigo positivo y el resto de grupos experimentales, $t(91)=2.12, p$ (una cola) $=$ $.013, R^{2}=.06$. Al ser el tamaño de efecto medio, es posible que el análisis carezca de toda la potencia estadística necesaria para llegar a afirmar con total certeza que los efectos aquí encontrados puedan darse en la población. Aun así, los datos encontrados son relevantes y deben ser tomados en cuenta.

\section{Discusión}

En el presente trabajo se pretende realizar una comparación entre diferentes métodos de corrección de error con el fin de hallar cuál de ellos es más adecuado para acelerar el aprendizaje de una tarea de discriminación condicional en diferentes grupos de edad. Para dar respuesta al planteamiento mencionado se propusieron una serie de hipótesis:

En la primera de ellas se planteó que la condición de corrección por seguimiento de regla sería más efectiva en los participantes de mayor edad que en los menores. Los resultados obtenidos han mostrado que no existen diferencias estadísticamente significativas, es decir, que el seguimiento de regla es igualmente eficaz en adultos de 19 - 20 años que en niños de 7-8.

Como se mencionó en la introducción, para que el seguimiento de una regla llegue a ser efectivo, es imprescindible que el individuo sea previamente entrenado en múltiples ejemplares. Teniendo en cuenta esto, los datos obtenidos podrían explicarse por la exposición de ambos grupos de edad a suficientes entrenamientos en seguimiento de regla a lo largo de su historia de aprendizaje como para poder responder de forma adecuada ante la nueva tarea presentada. 


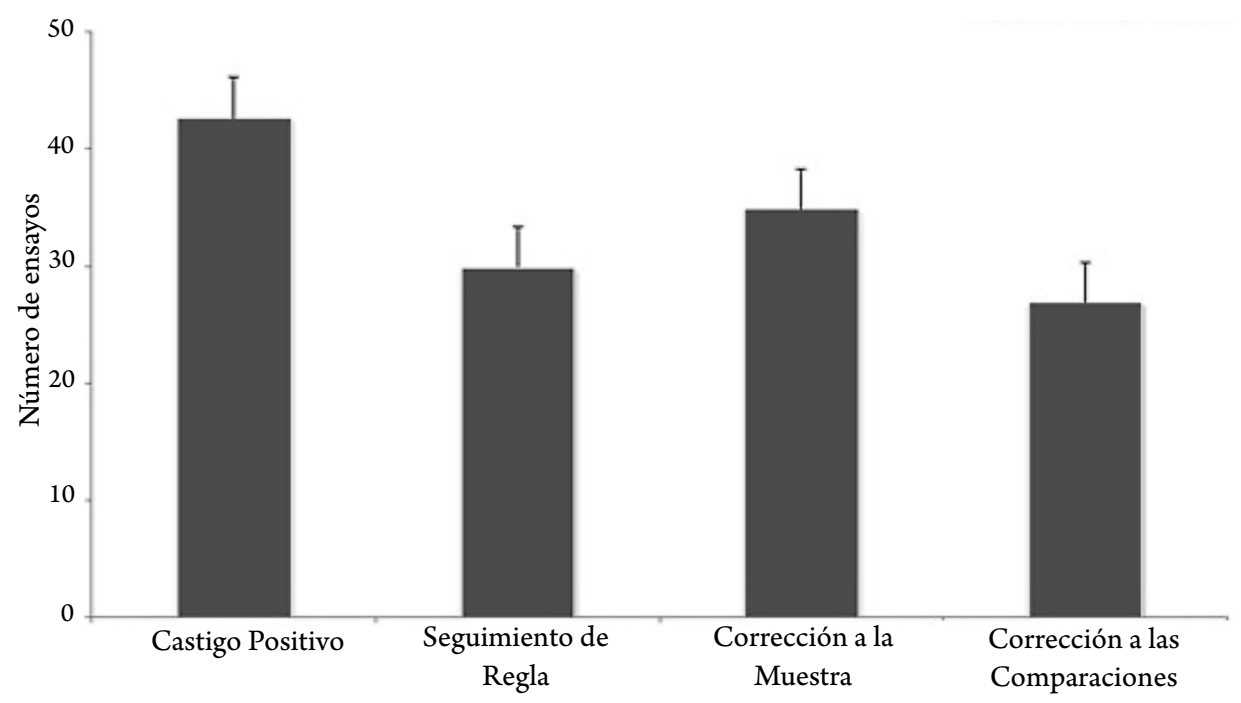

Figura 5. Número medio de ensayos necesarios para alcanzar el criterio de aprendizaje en los cuatro grupos experimentales. Hubo diferencias entre el grupo corregido con castigo positivo y los restantes.

Además, es necesario recordar que el término que aquí se entiende como seguimiento de regla, no se corresponde en su totalidad con el encontrado en la literatura científica. Por tanto, es posible que ambos sean funcionalmente distintos y no ejerzan el mismo efecto sobre los participantes. De esta forma, si se esperaba que los individuos de mayor edad tuviesen una mejor ejecución, esto no tendría por qué ser así con el seguimiento de regla no verbal.

De estas explicaciones se derivan varias líneas de investigación futura. Por una parte, resultaría de interés realizar un trabajo que estudie de forma sistemática la efectividad de la corrección con seguimiento de regla en diferentes edades, comparando adultos con niños más pequeños que los que han participado en esta investigación. Si se encontraran diferencias a favor de los adultos podría ser debido a una insuficiente presentación de ejemplares en los niños. Por otra parte y de manera aislada, también podría ser útil comparar el seguimiento de regla verbal y no verbal con el fin de determinar sus características y si la función de uno es equiparable a la del otro. Determinando las particularidades de cada procedimiento se podría seleccionar el método más conveniente para corregir errores en discriminaciones condicionales. 
La segunda hipótesis propuso que corregir con castigo positivo sería menos efectivo que el resto de correcciones. Los resultados obtenidos señalan que esto parece ser así, por lo que usar esta forma de corrección no es equiparable a las otras utilizadas (seguimiento de regla, corrección llevada a la muestra y corrección llevada a las comparaciones), acarreando una peor ejecución en los participantes a los que se les ha aplicado dicho procedimiento.

Pese a que este grupo necesita un número significativamente más elevado de ensayos, los participantes aprenden la tarea. Es por ello que puede entenderse que existan estudios sobre discriminaciones condicionales que usen el castigo positivo, ya que, en última instancia, se logra el aprendizaje. Sin embargo, no hay que olvidar que, además, el castigo presenta limitaciones y desventajas a largo plazo que, aparentemente, no se dan en el resto de procedimientos.

Es posible que en investigación los efectos temporales del castigo sean poco visibles, ya que lo que se observaría, sobre todo, es el producto inmediato de reducción de una conducta, pero no se contemplarían las repercusiones en un periodo de tiempo más amplio, aspecto que sí resulta más relevante en el ámbito aplicado, por ejemplo, el educativo.

Es por ello que se hace interesante la idea de analizar en otras investigaciones los efectos a largo plazo de la corrección con castigo, en comparación con otros métodos correctivos como los estudiados en este trabajo. Por ejemplo, administrando la prueba a los mismos participantes después de un tiempo prudencial, para determinar qué aprendizaje resulta más duradero.

Además, se podría estudiar por separado tanto las correcciones llevadas a la muestra y a las comparaciones, como el seguimiento de regla, indagando sobre la forma en la que estas correcciones pueden afectar a factores como la generalización del aprendizaje o la formación de clases de equivalencia. Asimismo, sería necesario esclarecer si el uso de estos procedimientos presenta limitaciones que hagan que la utilización de estas condiciones sea menos adecuada.

Resulta interesante comentar también que la ausencia de diferencias estadísticamente significativas entre las correcciones, que no son castigo positivo, puedan provenir del hecho de que al errar, los ensayos se repetían hasta que el participante emitía la respuesta correcta. Existen estudios cuyo procedimiento consiste en corregir haciendo que el participante repita el ensayo en el que ha fallado varias veces. Ejemplo de ello es el trabajo de Ollendrick, Matson, Esveldt-Dawson y Shapiro (1980), quienes compararon en varios estudios un tipo de corrección llamada práctica positiva con formas de corrección más tradicionales. Esta práctica consistía 
en hacer que el alumno repitiera cinco veces una serie de pasos al errar en una palabra escrita. Los datos mostraron que esta práctica combinada con el reforzamiento diferencial aplicado cuando acertaban daba mejores resultados que otra forma de corrección más tradicional.

También los estudios de Shumaker y Sherman (1970) y Nutter y Reid (1978) corrigen los errores con varias repeticiones del ensayo en cuestión (cuatro y tres veces respectivamente) obteniendo resultados satisfactorios en la ejecución de las tareas de sus respectivos experimentos.

De estos trabajos se derivan cuestiones interesantes que podrían ser estudiadas en el futuro: iSe obtendrían los mismos resultados si a los ensayos erróneos se les aplica una sobrecorrección?, ¿Hay condiciones de este experimento a las que les beneficia más que a otras la repetición de ensayos en los que se ha cometido un fallo?

Existen diferentes variables que se pueden combinar en la forma de corregir, haciendo más o menos complejo este proceso. Es necesario el estudio progresivo de cada una de ellas para poder determinar protocolos de actuación cada vez más depurados que ayuden en la optimización del tiempo invertido en la enseñanza mediante discriminaciones condicionales.

De forma aislada a las hipótesis de investigación, se han encontrado resultados que son de interés:

Por una parte, según los datos obtenidos, ni la edad ni la relación entre esta variable y los tipos de corrección ejercen influencia en la velocidad de adquisición de una discriminación condicional. Se podría concluir, pues, que el aprendizaje se va a producir a una rapidez similar, con independencia de los valores que se tomen de estas variables.

Aunque los resultados no lo avalen, se podría esperar que los participantes más pequeños, hayan sido expuestos a una menor cantidad y variedad de procedimientos de corrección, por lo que unos resultarían más efectivos que otros. Sin embargo, conforme la edad aumentase, estas diferencias tenderían a igualarse hasta que, en el grupo de mayor edad, no se encontrasen discrepancias entre la utilización de uno u otro procedimiento de corrección.

Normalmente, en la infancia es frecuente el uso de castigo positivo y no tanto de otro tipo de procedimientos más complejos. Debido a esto, cabría esperar que los participantes más pequeños respondieran con mayor efectividad ante los más familiares.

En personas de mayor edad, la experiencia en tareas de esta índole, probablemente, pueda ser más amplia, por lo que se presupone que también reciben un alto 
número de correcciones en sus diferentes formas. Tomando esto como cierto, a efectos prácticos, resultaría irrelevante corregir de uno u otro modo, ya que los participantes van a tener suficiente pericia en todas las condiciones como para acabar aprendiendo la tarea de forma rápida y eficaz.

A pesar de estas consideraciones, es posible que los participantes que conforman la muestra estudiada tengan suficiente experiencia como para responder de manera similar a uno u otro procedimiento. Por ello, en una posible investigación futura, podría ser interesante abarcar un rango de edad menor, para comprobar empíricamente si esta variable es un buen predictor de la susceptibilidad a los diferentes procedimientos de corrección.

Por otro lado, resulta imprescindible mencionar la diferencia encontrada entre el grupo control con respecto al resto de grupos que han recibido corrección.

Los resultados muestran que la ejecución cuando no se recibe retroalimentación ante los errores es mucho peor que cuando sí se proporciona corrección, sea del tipo que sea. Mientras que los participantes (independientemente de la edad) que han recibido retroalimentación de sus fallos alcanzan el criterio establecido, los participantes del grupo control bien no llegan a alcanzar el criterio, bien lo hacen, pero necesitan un número mucho más elevado de ensayos. Dentro de este grupo se observa que los participantes de mayor edad cometen más errores que el resto, lo cual podría deberse a que estos generen reglas erróneas sobre la prueba que interfieran en su correcta ejecución.

El no proporcionar retroalimentación de los fallos puede tener una serie de consecuencias si se está implementando un programa de reforzamiento intermitente, como, por ejemplo, de razón variable. En los programas de reforzamiento de este tipo, solo se recompensa al individuo tras la emisión de un número de respuestas determinado que va variando de ocasión en ocasión. Por tanto, hay situaciones en las que la conducta se produce de manera apropiada y no es reforzada, lo cual puede llevar a que el participante confunda esto con las respuestas que están siendo extinguidas por el programa. Siendo esto así, se puede ver ralentizado el aprendizaje de un contenido, además de que los ensayos correctos no reforzados pueden ejercer interferencia en pruebas posteriores (Valero \& Luciano, 1997).

Además, los resultados obtenidos pueden enmarcarse dentro del procedimiento de consecuencias diferenciales (PCD), formando una evidencia empírica más a favor de su beneficio. Para profundizar más en este tema, parte de los estudios realizados pueden consultarse en las revisiones de Goeters, Blakely y Poling (1992) y Fernández (2008). 
El PCD fue inicialmente usado en experimentos con animales no humanos. Entre ellos destaca el estudio realizado por Trapold (1970), que trabajó con diferentes grupos de ratas a las que administró consecuencias diferenciales.

Para su experimento utilizó 24 ratas, las cuales dividió en dos grupos principales: el experimental y el control. Todos los animales fueron entrenados para que respondieran pulsando la barra derecha cuando sonara un tono y a la barra izquierda cuando se oyera un clicker. A la mitad del grupo experimental se le reforzaba con comida ante el tono y sacarosa ante el clicker y al resto al contrario, mientras que al grupo control se le reforzaba con el mismo agente ante el tono o el clicker (a la mitad del grupo con comida y a la otra mitad con sacarosa).

Los resultados evidenciaron que el grupo reforzado de forma diferencial no solo aprendía más rápido, sino que la tasa de respuestas correctas era mayor y cometían menos errores.

El autor concluyó que el reforzamiento diferencial contribuía a generar en los individuos diferentes expectativas de reforzamiento y esto ayudaba a responder de forma más precisa, desembocando todo en una mayor velocidad de aprendizaje en este tipo de tareas.

Siguiendo esta lógica, se puede concluir que en el presente trabajo ha ocurrido un efecto similar entre el grupo control y los que recibieron corrección. En los grupos experimentales, las consecuencias ante el error eran más discriminables, por tanto, no sería desacertado afirmar que el PCD ha ejercido una influencia facilitadora del aprendizaje es estos grupos.

El conjunto de resultados provenientes de la presente investigación ha sido obtenido mediante un experimento cuya tarea fue una discriminación condicional de primer orden, con cuatro comparaciones y demora 0 . Sin embargo, las discriminaciones condicionales se caracterizan por una gran riqueza procedimental, pudiendo variar muchos elementos de ésta, como las comparaciones, el tipo de igualación, los reforzadores, etc. (García \& Benjumea, 2002). Por lo tanto, los datos que se han obtenido bajo unas circunstancias concretas pueden cambiar si éstas lo hacen. De ello deriva el interés de investigar si estos mismos resultados se hubiesen obtenido en caso de utilizar otro tipo de discriminación condicional o se hubieran aplicado diferentes modalidades sensoriales en los estímulos presentados.

Aun así, los datos hallados ofrecen unos resultados con unas aplicaciones directamente relacionadas con el ámbito práctico, donde es importante especificar qué consecuencias van a ser útiles si se quiere fortalecer o debilitar una conducta determinada. Los datos obtenidos pueden ser extrapolables a otros contextos de 
aprendizaje, sirviendo de guía y apoyo a otros profesionales, ayudándoles a economizar el tiempo invertido en la enseñanza de una tarea de este tipo al alumnado.

También, los resultados encontrados pueden ser de gran utilidad en investigación. Al conocerse ciertos aspectos que mejoran la velocidad de adquisición de una discriminación condicional, se pueden acelerar las investigaciones que se realicen sobre este campo. A pesar de las ventajas que esta investigación pueda tener, no hay que olvidar que los beneficios comentados hasta ahora son potenciales, ya que este estudio presenta una serie de limitaciones que hay que considerar.

Por un lado, se ha contado con la colaboración de 96 personas, un número ciertamente elevado, pero teniendo en cuenta las condiciones experimentales que se han contemplado, 20 en total, la cantidad de participantes por condición se ha visto reducida. Esto ha podido afectar al estudio de forma que, al realizar los análisis, no se hayan encontrado diferencias entre algunos grupos debido a que el conjunto de datos recabados ha sido insuficiente como para detectar diferencias y conformar una muestra representativa de la población diana. Aumentando el número de participantes, se podría conseguir una visión más fiable y válida de los efectos que los diferentes tipos de corrección pueden tener en el aprendizaje de discriminaciones condicionales.

Por último, es conveniente recordar que la muestra de este trabajo se ha compuesto en su totalidad de participantes de desarrollo típico, con lo que los resultados de esta investigación y su aplicabilidad se limitan a una población de este tipo. Pese a que los mecanismos que subyacen el aprendizaje son los mismos, es posible que haya poblaciones en las que sean necesarias ciertas consideraciones a la hora de elegir el procedimiento de corrección que mejor se adecue a las características de los participantes. Por ello, los resultados de esta investigación serían difícilmente extrapolables a individuos de desarrollo no típico. Por consiguiente, una posible vía de estudio futuras consistiría en la replicación de este estudio con población con discapacidad intelectual o trastorno del espectro autista, de forma que se amplíe el marco de aplicación práctica de los conocimientos derivados de la presente investigación.

Para finalizar, y a modo de conclusión, se pueden destacar varios aspectos. En primer lugar, se encontraron diferencias estadísticamente significativas entre el grupo control y los grupos experimentales, observándose una mejora en la ejecución de la tarea cuando en ésta se recibe retroalimentación ante los errores. Esto resalta la importancia de aplicar corrección ante los fallos si se quiere facilitar de manera relevante el aprendizaje de una tarea de igualación a la muestra. Además, los datos 
encontrados constituyen una evidencia empírica más a favor de la efectividad del PCD.

Por otra parte, dentro de los diferentes tipos de corrección, los datos evidenciaron que el castigo positivo es el procedimiento que arroja peores resultados, ya que parece ser que ralentiza el aprendizaje con respecto a otros métodos de corrección. Con base en esto y teniendo en cuenta la información existente en la literatura científica sobre los efectos perjudiciales y limitados del castigo positivo, sería más acertado utilizar como alternativa, cualquiera de las otras formas de corrección planteadas en este estudio.

Asimismo, los resultados obtenidos en la comparación en seguimiento de regla entre los participantes mayores y menores no fueron estadísticamente diferentes, por lo que esta hipótesis no se pudo validar. Es posible que la ejecución fuera similar porque ambos grupos estuvieran suficientemente entrenados en ese procedimiento de corrección. Además, es necesario investigar en profundidad sobre el seguimiento de regla verbal y no verbal para averiguar si ambos conceptos son equiparables o no y poder determinar, en última instancia, si los resultados obtenidos son debidos a que se han tomado como iguales dos términos diferentes.

Por último, se encontró que los diferentes grupos de edad se comportan de manera similar ante los procedimientos de corrección aplicados. A pesar de ello, puede ser esperable hallar diferencias según la edad, debido a la cantidad y variedad de procedimientos a los que pueden estar sometidos estos grupos a lo largo de su historia de aprendizaje. Es posible que la ausencia de diferencias esté debida al reducido tamaño de la muestra, por lo que, si en futuras investigaciones se alcanzara la significación estadística, esto podría ayudar a ajustar la enseñanza lo máximo posible a las características de los individuos.

\section{Referencias}

Barón, A., \& Galizio, M. (1990). Control de la conducta operante humana por medio de instrucciones. En E. Ribes y P. Harzem (Eds.), Lenguaje y Conducta (pp. 123-168). México: Trillas.

Constantine, B., \& Sidman, M. (1975). Role of naming in delayed matching-to-sample. American Journal of Mental Deficiency, 79, 680-689.

Fernández, M. A. (2008). El efecto de consecuencias diferenciales como herramienta útil para mejorar el aprendizaje discriminativo en humanos. Almería: Universidad de Almería. 
Flores, C., \& Bruner, C. (2005). Efecto del intervalo entre reforzadores sobre el índice de discriminación en tareas de igualación a la muestra. Acta Comportamentalia, 13, 87-97.

García, A. (2012). Aprendizaje complejo. Sevilla: San Bernardo.

García, A., \& Benjumea, S. (2002). Orígenes, Ampliación y Aplicaciones de la Equivalencia de Estímulos. Apuntes de Psicología, 20, 171-186.

García, A., \& Benjumea, S. (2006). Discriminación condicional de la propia conducta en palomas: el papel de la longitud de la conducta-muestra. International Journal of Psychology and Psychological Therapy, 6, 331-342.

García, A., Pérez, F., Martín, R., Gutiérrez, M. T., Benjumea, S., Gómez, J., \& Pérez, V. (2011). Efecto de la edad y el tipo de reforzador en la equivalencia-equivalencia mediante un procedimiento de partición. International Journal of Psychological Research, 4, 7-15.

Goeters, S., Blakely, E., \& Poling, A. (1992). The differential outcomes effect. The Psychological Record, 42, 389-411.

Gómez, J., García, A., \& Pérez, V. (2014). Failure to find symmetry in pigeons after multiple exemplar training. Psicothema, 26, 435-441.

Greer, R. D., \& Ross, D. E. (2014). Análisis de la conducta verbal. Cómo inducir y expandir nuevas capacidades verbales en niños con retraso en el lenguaje. Madrid: Grupo 5.

Hurtado, C., Robayo, M. A., \& Peña, T. E. (2007). Efectos en la ejecución durante una tarea de igualación a la muestra según el tipo y orden de exposición a las pruebas de transferencia. Revista Javeriana, 6, 425-440.

Islas, A., \& Flores, C. (2007). Papel de la retroalimentación en la adquisición y transferencia de discriminaciones condicionales en estudiantes universitarios. Enseñanza e Investigación en Psicología, 12, 65-77.

Kazdin, A. E. (1994). Behavior modification in applied settings. California: Brooks

Martínez, H., Ortiz, G., \& González, A. (2007). Efectos diferenciales de instrucciones y consecuencias en ejecuciones de discriminación condicional humana. Psicothema, 19, 14-22.

Martínez, H., \& Tamayo, R. (2005). Interactions of contingencies, instructional accuracy, and instructional history in conditional discrimination. The Psychological Record, 55, 633-646.

Nutter, D., \& Reid, D. (1978). Teaching retarded women a clothing selection skill using community norms. Journal of Applied Behavior Analysis, 11, 475-487. 
Ollendrick, T. H., Matson, J. L., Esveldt-Dawson, K., \& Shapiro, E. S. (1980). Increasing spelling achievement: an analysis of treatment procedures utilizing an alternating treatments design. Journal of Applied Behavior Analysis, 13, 645-654.

Ortega, M., Pacheco, V., Sánchez, E., \& Rivera, D. A. (2012). Effect of different feedback modalities in the intelligent behavior of elementary students. Enseñanza e Investigación en Psicología, 17(1), 137-149.

Ortiz, G., \& Cruz, Y. (2011). El papel de la precisión instruccional y la retroalimentación en la ejecución y descripciones postcontacto. Revista Mexicana de Análisis de la Conducta, 37(1), 69-87. doi:10.5514/rmac.v37.i1.24740

Pérez, L. A. (2001). Procesos de aprendizaje de discriminaciones condicionales. Psicothema, 13(4), 650-658.

Pérez, V. (2015). Clases de equivalencia y conducta verbal. Conductual, 3(1), 26-44. Quesada, J., García, M., Ruiz, M., \& García, A. (2015). Influencia de las etiquetas verbales en una situación de elección de recompensas. En A. García (presidencia), IV Congreso de la Sociedad para el Avance del Estudio Científico del Comportamiento. Congreso llevado a cabo en Córdoba.

Ribes, E., \& Martínez, H. (1990). Interaction of contingences and rule instructions in the perfomance of human subjects in condicional discrimination. The Psychological Record, 40, 565-586.

Rodgers, T. A., \& Iwata, B. A. (1991). An analysis of error-correction procedures during discrimination training. Journal of Applied Behavior Analysis, 24, 775-781.

Rodríguez, M. E., Ribes, E., Valencia, L. M. T., \& González, L. F. (2011). Efecto de un entrenamiento observacional con descripciones en la transferencia extradimensional. Revista Mexicana de Análisis de la Conducta, 37(2), 155-175. doi:10.5514/rmac.v37.i2.26145

Rodríguez, M. T., García, A., Gutiérrez, M. T., Pérez, V., \& Bohórquez, C. (2009). Competencia entre estímulos condicionales propioceptivos y exteroceptivos en una tarea de discriminación condicional. Psicothema, 21(3), 390-396.

Serrano, M. A., \& Montes, E. E. (2014). Efectos de las relaciones de condicionalidad en tareas de igualación a la muestra. Journal of Behavior, Health \& Social Issues, 6(1), 67-78. doi:10.5460/jbhsi.v6.1.47603

Shumaker, J., \& Sherman, J. A. (1970). Training generative verb usage by imitation and reinforcement procedures. Journal of Applied Behavior Analysis, 3, 273-287. Sidman, M. (1971). Reading and auditory-visual equivalences. Journal of Speech and Hearing Research, 14, 5-13. 
Sidman, M. (2000). Equivalence relations and the reinforcement contingency. Journal of the Experimental Analysis of Behavior, 74, 127-146.

Sidman, M., \& Cresson, O. (1973). Reading and crossmodal transfer of stimulus equivalences in severe retardation. American Journal of Mental Deficiency, 77( 5), 515-523.

Sidman, M., \& Tailby, W. (1982). Conditional discrimination vs. matching to sample: an expansion of the testing paradigm. Journal of the Experimental analysis of Behavior, 37, 5-22.

Skinner, B. F. (1938). The Behavior of organisms. New York: Appleton-Century-Crofts.

Skinner, B. F. (1953). Science and human behavior. New York: Macmillan (Trad. castellana: Barcelona, Fontanella, 1977).

Thorndike, E. L. (1906). Principles of teaching. New York: Seil

Törneke, N., Luciano, M. C., \& Valdivia, S. (2008). Rule-Governed Behavior and Psychological Problems. Journal of Psychology and Psychological Therapy, 8(2), 141-156.

Trapold, M. A. (1970). Are expectancies based upon different positive reinforcing events discriminably different? Learning and Motivation, 1, 129-140.

Valero, L., \& Luciano, M. C. (1997). Discriminación condicional en niños: Los efectos de contingencias diferenciales de reforzamiento/tiempo fuera. Psicothema, 9(3), 599-608.

Villanueva, S., Mateos, R., \& Flores, C. (2008). Efectos del contenido y distribución de la retroalimentación sobre la discriminación condicional de segundo orden. Acta Comportamentalia, 16(2), 211-221.

Worsdell, A. S., Iwata, B. A., Dozier, C. L., Johnson, A. D., Neidert, P. L., \& Thomason, J. L. (2005). Analysis of response repetition as an error-correction strategy during sight-word reading. Journal of Applied Behavior Analysis, 38, 511-527.

Recibido Marzo 3, 2016 /

Received March 3, 2016

Aceptado Febrero 18, 2017 /

Accepted February 18, 2017 